\title{
Tortuosidade e conectividade da rede trabecular do rádio distal a partir de imagens microtomográficas
}

\author{
Waldir Leite Roque*, Katia Arcaro, Ricardo Bigolin Lanfredi
}

Resumo A osteoporose é uma doença que causa perda de massa óssea e deterioração da estrutura trabecular. A rede formada pelo osso trabecular é muito importante para estabelecer a competência mecânica do esqueleto. Estudos mostram que a microarquitetura trabecular pode elevar a predição do risco de fratura para cerca de $90 \%$, por isso a sua investigação é de grande interesse da comunidade científica. Vários parâmetros estão relacionados à competência mecânica da estrutura óssea, entretanto, a tortuosidade trabecular ainda não foi seriamente levada em consideração e sua contribuição é desconhecida. Neste trabalho, o enfoque está direcionado à estimativa da tortuosidade trabecular e sua correlação com a conectividade, através da característica de Euler-Poincaré, para amostras do osso rádio distal in vitro, a partir de imagens de microtomografia computadorizada. Um método para estimar a tortuosidade do volume trabecular é apresentado e a tortuosidade é computada para os dois sentidos das três direções do volume. Os resultados apresentam valores distintos da tortuosidade dependendo da qualidade da rede trabecular e uma correlação positiva $(r=0,64)$ com a característica de Euler-Poincaré; além disso, observa-se que a tortuosidade busca alinhar-se nas direções de maiores tensões e compressões imprimidas à rede trabecular. Tais resultados evidenciam a importância da tortuosidade como parâmetro estrutural da rede trabecular.

Palavras-chave Osso trabecular, Tortuosidade, Característica de Euler-Poincaré, Competência

Mecânica, Osteoporose.

\section{Trabecular network tortuosity and connectivity of distal radius from microtomographic images}

\begin{abstract}
Osteoporosis is disease causing bone mass loss and deterioration of the trabecular structure. The trabecular network is very important to establish the mechanical competence of the skeleton. It is known that the trabecular microarchitecture can improve the fracture risk prediction up to 90\%, for this reason the trabecular bone investigation has become of great interest for the scientific community. Several parameters provide structural details of the bone mechanical competence, however, the trabecular network tortuosity has not been seriously taken into account yet and its contribution is unknown. This paper directs attention to the trabecular tortuosity estimation and its correlation to the connectivity, through Euler-Poincaré characteristic, from in vitro distal radius bone samples by micro-computed tomographic images. A method to estimate the trabecular bone tortuosity is presented and its value is computed for both directions of the three volume axis. The results indicate that the trabecular tortuosity depends on the trabecular network quality and a positive correlation $(r=0.64)$ with the Euler-Poincaré characteristic. Inasmuch, it has been observed that the tortuosity tends to get aligned in the direction of main stress imposed on trabecular bone network. These results support the tortuosity as an important structural parameter.
\end{abstract}

Keywords Trabecular bone, Tortuosity, Euler-Poincaré characteristic, Mechanical Competence, Osteoporosis. 


\section{Extended Abstract}

\section{Introduction}

Osteoporosis is a disease characterized by bone mass loss and deterioration of the trabecular structure. A consequence of that is an increase of fractures, leading to the need of treatment with very high cost for both public and private health care. It is known that the bone mineral density (BMD) is responsible for 70-80\% of the bone strength (Homminga et al., 2003); however recent studies show that it can be raised up to $90 \%$ due to the trabecular microarchitecture (Accardo et al., 2005; Carbonare et al., 2005). As BMD is a global measure, it is not able to detect fragilities in the trabecular structure.

The cancellous bone is essentially composed of trabecular bone network and marrow. With current development of imaging, the study of the trabecular structure became feasible and measurable. The cancellous bone can be modeled as a twophase porous media, where the trabeculae are the solid phase and marrow the soft phase. The tortuosity of the trabeculae seems to play an important role to the mechanical competence of the structure, in other words to the bone strength capacity to load, nevertheless up to now no study has been done to estimate the trabecular network tortuosity and its relation to other structural parameters. In this paper the trabecular tortuosity is estimated and its correlation to the Euler-Poincaré characteristic is analyzed.

\section{Material and Methods}

The work is based on $3 D$ micro-computed tomography $(\mu C T)$ images of distal radius trabecular bone from 15 cadavers. The samples were harvested about $10 \mathrm{~mm}$ from the endplate of the radius and scanned with a $\mu C T-20$ scanner considering an isotropic voxel size of $34 \mu \mathrm{m}$. The $3 D \mu C T$ images were filtered with a $3 D$ Gaussian filter. In each case the gray-level histograms of filtered images contained two peaks corresponding to marrow and bone; thus, the images were binarized, using a global threshold equal to the minimum between the peaks. The craniocaudal direction was identified with the z-axis. Details concerning the samples preparation and image protocols are in Laib et al. (2001). The tortuosity and connectivity studies were done for 15 individual sets with $239 \mu$ CT images in each. Geometrically, tortuosity is defined as $\tau=L_{G} / L_{E}$, where $L_{G}$ is the geodesic distance between two connected points and $L_{E}$ is the Euclidian distance between these points. The tortuosity is estimated applying the geodesic reconstruction algorithm (Gommes et al., 2009; Roque et al., 2011) and the Euler-Poincaré characteristic is estimated based on the algorithm discussed in (Vogel and Kretzshmar, 1996) and are provided in Tables 1 and 2.

\section{Results and Discussion}

Trabecular tortuosities were estimated for the 15 samples considering a sweeping plane in each one of the six directions, with the results given in Table 1. From these, it is noticed that the tortuosity can differ significantly between positive and negative directions for the same sample. This is a consequence of the trabecular network connectivity in one direction be different from the other, but also due to the plateness/rodness distribution of the trabeculae in the sweeping direction.

Defining the directional tortuosity deviation (DTD) as $\Delta \tau_{k}=\left|\tau_{+k}-\tau_{-k}\right|$, where $k=x, y, z$, and $\sigma=\sqrt{\Delta \tau_{x}{ }^{2}+\Delta \tau_{y}{ }^{2}+\Delta \tau_{z}{ }^{2}}$, it is possible to analyze the tortuosity variation according to the direction and the sample. For sample 269 in the $x$ direction $\Delta \tau_{x}=0.1406$, while for the sample 273 in the $z$ direction $\Delta \tau_{z}=0,0001$. These are the maximum and minimum DTDs from all samples. From DTD analysis, it can be seen that the trabeculae present a preferred orientation with respect to the sweeping plane direction. In addition, for each sample, the shortest DTD occurs in the $z$ direction, which is the distal-proximal radius direction. This indicates that the trabeculae get aligned in the direction of high stress (Tabor, 2009). It is observed a positive correlation $(r=0.64)$ between Euler-Poincaré characteristic (CEP) and $\sigma$. Inasmuch, from the volumetric representation of the samples 266, 268 and 269 (Figures 3a-c) and their tortuosities, it is noticed that as lower the connectivity, higher the DTDs, as a consequence of much larger cavities. This can be observed when a single trabecula is considered for the samples 266 and 269 (see Figures 4a,b).

\section{Conclusion}

In this paper the trabecular tortuosities and Euler-Poincaré characteristics were estimated for $15 \mu C T$ image samples of distal radius bone. It has been shown that the tortuosity of the trabecular network differs from sample to sample and even in the positive and negative directions of a single sample, according to the connectivity and/or the plateness/rodness distribution. In addition, it has been observed that the tortuosity increases for lower connected trabecular network due to the fact that low connectivity is accompanied by a higher number and size of cavities. The correlation coefficient between CEP and $\sigma$ was found to be $r=0.64$.

The results here are novel in the sense that there is no previous trabecular bone tortuosity estimation and its correlation with CEP presented in the literature. Nevertheless, further studies have to be developed to better understand the role that tortuosity plays to the mechanical competence of the trabecular structure. 


\section{Introdução}

Com o aumento da longevidade, a incidência da osteoporose vem se tornando um problema não apenas de natureza clínica, mas também de natureza socioeconômica. A principal consequência da osteoporose é a ocorrência de fraturas e fissuras ósseas que, além de causar desconforto, necessitam de tratamentos e internações hospitalares, contribuindo, muitas vezes, para a degradação da saúde do paciente, aumentando a morbidade e podendo levá-lo a óbito. Todo este processo envolve custos elevados, quer do setor público, quer do setor privado, sendo projetado em 25 bilhões de dólares os custos com a osteoporose nos EUA para 2025 (Burge et al., 2007). No Brasil, dados indicam que um em cada três pacientes com fratura no osso ilíaco são diagnosticados como tendo osteoporose e, entre estes, apenas um em cada cinco recebem algum tipo de tratamento. Os custos para o sistema de saúde privado no Brasil, apenas com a fratura osteoporótica do fêmur, foi da ordem de 12 milhões de reais ( 7,5 milhões de dólares) no período de um ano, entre julho de 2003 e junho de 2004 (Araújo et al., 2005). Neste sentido, torna-se, de fato, muito importante que se desenvolvam técnicas que possam auxiliar a predição do risco de fratura de um paciente de forma não invasiva e a um custo moderado.

A osteoporose é uma enfermidade osteometabólica que leva à perda de massa óssea e deterioração da estrutura do osso canceloso. O osso canceloso é essencialmente formado por uma rede de trabéculas e pela medula, sendo envolvido por uma parede densa chamada osso cortical. Por ser um tecido metabolicamente mais ativo, o osso trabecular é mais vulnerável do que o osso cortical. O padrão ouro para o diagnóstico da osteoporose é a densitometria mineral óssea (DMO), que é responsável por cerca de 70-80\% da variação da resistência óssea (Homminga et al., 2003); por outro lado, estudos recentes indicam que a microarquitetura trabecular eleva esse valor para aproximadamente 90\% (Accardo et al., 2005; Carbonare et al., 2005). Em geral, valores baixos de DMO sinalizam risco de fratura, porém alguns pacientes com valores considerados moderados de DMO apresentam quadros de traumas ósseos graves (Homminga et al., 2003; Wesarg et al., 2011). Como a DMO é uma medida que engloba toda a estrutura óssea (osso cortical mais osso canceloso), esta não é capaz de detectar as deficiências e fragilidades inerentes à estrutura do osso trabecular.

Nos últimos anos a evolução de recursos tecnológicos para exames por imagem permitiu que características histomorfométricas e geométricas/topológicas da estrutura trabecular, como a fração volumétrica, espessura trabecular, conectividade, propriedades elásticas etc., pudessem ser amplamente exploradas e a microtomografia computadorizada $(\mu \mathrm{CT})$ ampliou ainda mais este horizonte. Um trabalho recente (Roque et al., 2010) mostra uma correlação positiva entre a fração volumétrica de osso trabecular, a conectividade da rede trabecular, estimada pela característica de Euler-Poincaré, e o módulo de Young, para sua elasticidade; esses parâmetros desempenham um papel importante para a competência mecânica da estrutura óssea, em outras palavras a resistência da estrutura quando sujeita a cargas. Por outro lado, a estrutura trabecular pode ser vista como composta por treliças que proporcionam a resistência mecânica da estrutura quando sujeita a tensões. Porém estas treliças apresentam-se de forma sinuosa, devendo tal característica ser levada em consideração quando da estimativa da resistência mecânica do osso trabecular.

Além disso, o osso canceloso assemelha-se a um meio poroso formado basicamente por uma fase sólida, composta pelo osso trabecular, e uma fase fluida, composta pela medula. É comum no estudo da estrutura trabecular in vitro fazer um tratamento preliminar de modo a retirar a medula, deixando apenas as cavidades medulares. Em geral, em um meio poroso com duas fases, a fase sólida é denominada grão e a fase das cavidades é denominada poro (Clennell, 1997).

Em meios porosos, a tortuosidade $\tau$ é uma medida do grau de sinuosidade de um poro, entretanto a tortuosidade geométrica é um conceito definido para uma estrutura filamentosa qualquer. A rede trabecular apresenta-se de forma sinuosa, devendo existir uma relação entre sua tortuosidade e os demais parâmetros estruturais. A literatura da área ainda não dispõe de um estudo sistemático sobre a tortuosidade trabecular, em particular a partir de imagens tomográficas, por isso sua contribuição para a competência mecânica ainda é pouco conhecida. Alguns trabalhos recentes discutem a importância da tortuosidade da cavidade medular para a estrutura do osso canceloso, utilizando técnicas de ultrassom para estimá-la em algumas amostras in vitro de ossos bovino e humano (Attenborough et al., 2005; Aygün et al., 2009; Hughes et al., 2007).

Neste trabalho, é apresentado e aplicado um método para estimar a tortuosidade da rede trabecular, a partir de imagens obtidas por microtomografia computadorizada, para um conjunto de 15 amostras do osso rádio distal e, em seguida, são discutidos alguns aspectos relativos à tortuosidade e à conectividade trabecular. 


\section{Materiais e Métodos}

O estudo deste trabalho está baseado em imagens 3D geradas por $\mu \mathrm{CT}$ do osso trabecular do rádio (região distal), as quais foram obtidas in vitro de 15 cadáveres. As amostras, com tamanho lateral de $12 \mathrm{~mm}$, foram coletadas a uma distância média de $9,75 \mathrm{~mm}$ a partir da extremidade distal do rádio. Foram selecionadas regiões de interesse (ROI) com tamanhos que variam de acordo com aspectos clínicos do material e as imagens foram obtidas com o scanner microCT-20 (Scanco Medical, Brüttisellen, Switzerland) com um voxel isotrópico de $34 \mu \mathrm{m}$ de lado. Para remoção de ruído, as imagens $3 \mathrm{D} \mu \mathrm{CT}$ foram filtradas com uso de um filtro Gaussiano 3D. Em cada caso, os histogramas em escalas de cinza das imagens filtradas continham dois picos, correspondendo à medula e ao osso; as imagens foram, então, binarizadas usando um limiar global igual ao mínimo entre os dois picos. A direção crânio-caudal foi identificada com o eixo $z$ de cada amostra. Detalhes adicionais com respeito à preparação das amostras e protocolos de aquisição das imagens estão descritos no trabalho de Laib et al. (2001).

Nesse trabalho, foram considerados 15 conjuntos de imagens $\mu \mathrm{CT}$, tomando-se 239 fatias em cada conjunto, com ROIs formadas por $212 \times 212$, $237 \times 237,242 \times 242,252 \times 252$ e $257 \times 257$ pixels; as 10 outras possuem $268 \times 268$ pixels. As matrizes binárias das imagens e o cálculo da característica de Euler-Poincaré foram realizados com o programa OsteoImage (Roque et al., 2007), o qual vem sendo desenvolvido pelos autores especialmente para a análise de imagens trabeculares. As figuras com imagens tridimensionais presentes neste trabalho foram geradas com os softwares OsteoImage e ImageJ.

\section{Tortuosidade}

Em um meio poroso a tortuosidade $\tau$ desempenha um papel importante na descrição da estrutura e apresenta distintos conceitos (Clennell, 1997). Entretanto, matematicamente, a tortuosidade caracteriza a sinuosidade de um filamento. Neste trabalho, este conceito é aplicado às trabéculas. Geometricamente, a tortuosidade $\tau$ é definida como

$$
\tau=\frac{L_{G}}{L_{E}}
$$

onde $L_{G}$ é a distância geodésica entre dois pontos conexos na rede trabecular, e $L_{E}$ é a distância Euclidiana entre eles. Na literatura, alguns autores consideram a distância Euclidiana na Equação 1, como sendo a distância em linha reta entre dois pontos do poro, independente da geometria das fases. No entanto, quando o espaçamento entre os planos de varredura são pequenos, a distância Euclidiana entre os pontos e a distância Euclidiana entre os planos paralelos que delimitam a estrutura podem ser considerados aproximadamente iguais; neste caso, é comum considerar $L_{E}$ como a distância entre os planos (Wua et al., 2006), como ilustrado na Figura 1.

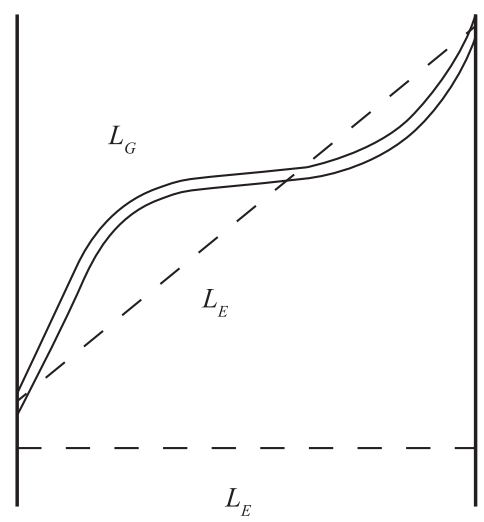

Figura 1. Ilustração da distância geodésica $L_{G}$ e da distância Euclidiana $L_{E}$, tomada entre planos e entre pontos.

Figure 1. Geodesic distance $L_{G}$ and Euclidian distance $L_{E}$, taken between planes and points.

O cálculo da tortuosidade sobre o espaço imagem requer o desenvolvimento de algoritmos que calculem a tortuosidade com base nos pixels ou voxels que o representam. A técnica adotada neste trabalho está fundamentada no princípio da reconstrução geodésica inicialmente proposta em Gommes et al. (2009), que busca associar um valor de tortuosidade para toda a rede de poros.

Neste trabalho, a técnica consiste essencialmente em considerarmos uma imagem 3D da estrutura trabecular e um sentido de referência, digamos, na direção positiva do eixo $z$, denominada $+z$. A estrutura é varrida por um plano normal ao sentido $+z$ e, utilizando-se o procedimento da RG, estima-se a distância geodésica $L_{G}$ de uma trabécula, a partir do plano varredor inicial até uma fatia $m$, sendo o número $n$ de RGs necessárias para alcançar a $m$-ésima fatia. As RGs recobrem todos os voxels que correspondem as trabéculas que são conexas as da fatia inicial. Por outro lado, a distância Euclidiana $L_{E}$ é determinada pelo número de fatias obtidas pela propagação paralela do plano varredor. O número $n$ de RGs necessárias para recobrir todas as trabéculas conexas até a $m$-ésima fatia é tal que $m \leq n$, com a igualdade ocorrendo apenas quando a rede trabecular é perpendicular ao plano varredor.

Como para uma dada distância Euclidiana $L_{E}(\mathrm{~m})$ tem-se diversos valores de distâncias geodésicas, 
já que são necessárias mais reconstruções do que o número de fatias para recobrir a rede trabecular até a fatia $m$, temos, portanto, uma distribuição $\rho\left(L_{E}, L_{G}\right)$ de distâncias Euclidianas e geodésicas, onde $\rho d L_{G} d L_{E}$ é proporcional ao número de pontos com distância Euclidiana $\left[L_{E}, L_{E}+d L_{E}\right]$ e distância geodésica $\left[L_{G}, L_{G}+d L_{G}\right]$. A Figura 2a ilustra uma distribuição para toda extensão na direção $+z$ para a amostra 266. Para estimar a tortuosidade da rede trabecular, define-se $\left\langle L_{G}(m)\right\rangle$ como a distância geodésica média para uma certa distância Euclidiana $L_{E}(\mathrm{~m})$. $\mathrm{O}$ gráfico de dispersão dos pontos $\left(L_{E}(m),\left\langle L_{G}(m)\right\rangle\right)$ pode ser facilmente obtido a partir das imagens e, assim, a tortuosidade pode ser estimada da equação (1) como a inclinação da reta de melhor ajuste para a distribuição das distâncias, como é apresentado na Figura $2 \mathrm{~b}$.

Em (Roque et al., 2011) o algoritmo da RG é detalhado e aplicado a imagens bi e tridimensionais. Em princípio, para imagens tridimensionais, o
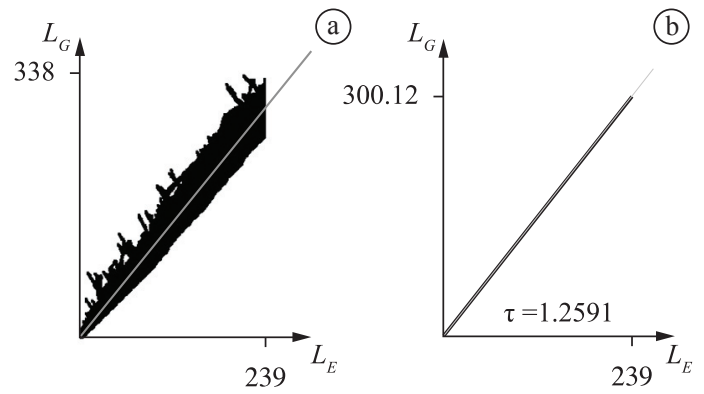

Figura 2. a) distribuição de distâncias; $b) \mathrm{L}_{E} \mathrm{x}\left\langle\mathrm{L}_{G}\right\rangle$, tortuosidade $\tau_{+z}$ para a amostra 266.

Figure 2. a) distance distribution; b) $\mathrm{L}_{E} \times\left\langle\mathrm{L}_{G}\right\rangle$, tortuosity $\tau_{+z}$ to the sample 266. procedimento da $\mathrm{RG}$ poderia considerar também elementos estruturantes com 18 ou 26-vizinhos $\left(E E_{18}\right.$ ou $\left.E E_{26}\right)$. No entanto, tais elementos estruturantes geram RGs que são bastante suscetíveis à limiarização e por isso não foram adotados. Aplicando a técnica da RG para o cálculo da tortuosidade, a Tabela 1 apresenta os valores obtidos nos seis sentidos de varredura para cada uma das 15 amostras.

\section{Conectividade}

A característica de Euler-Poincaré (CEP) é uma técnica que vem sendo utilizada para estimar a conectividade da rede trabecular e pode ser calculada a partir de pares consecutivos de imagens igualmente espaçadas, chamados disectors, os quais delimitam um volume da estrutura sob análise (Gundersen et al., 1993; Roberts et al., 1997; Vogel e Kretzshmar, 1996). A CEP de uma coleção de objetos $3 \mathrm{D}$ pode ser calculada por $\mathrm{CEP}=\# \mathrm{I}-\# \mathrm{~B}+\# \mathrm{H}$, onde $\# \mathrm{I}$ é o número de partes isoladas, \#B é o número de ramificações e \#H é o número de cavidades fechadas.

Vogel e Kretzshmar (1996) apresentam a técnica aqui utilizada e implementada em (Roque et al., 2009), para a contagem de \#I, \#B e \#H automaticamente em um volume de um meio poroso a partir das imagens binárias do disector. Quanto mais conectada se apresenta a estrutura, menores e negativos são os valores da CEP (Chappard et al., 1999).

Tendo estimado o valor da CEP em cada disector de uma amostra, a soma dos valores em todos eles fornece uma estimativa do número de conexões presentes na estrutura. Como as amostras utilizadas têm volumes distintos, pode-se normalizar os resultados dividindo-os

Tabela 1. Tortuosidades, DTDs e $\sigma$ estimados para as 15 amostras.

Table 1. Tortuosities, DTDs and $\sigma$ estimated to the 15 samples.

\begin{tabular}{ccccccccccc}
\hline Amostra & $\tau_{+z}$ & $\tau_{-z}$ & $\Delta \tau_{z}$ & $\tau_{+y}$ & $\tau_{-y}$ & $\Delta \tau_{y}$ & $\tau_{+x}$ & $\tau_{-x}$ & $\Delta \tau_{x}$ & $\boldsymbol{\sigma}$ \\
\hline 254 & 1,2895 & 1,2779 & 0,0116 & 1,4726 & 1,4569 & 0,0157 & 1,6514 & 1,6847 & 0,0333 & 0,0386 \\
255 & 1,2618 & 1,2749 & 0,0131 & 1,4693 & 1,4556 & 0,0137 & 1,6534 & 1,6137 & 0,0397 & 0,0440 \\
256 & 1,2711 & 1,2807 & 0,0096 & 1,5870 & 1,5317 & 0,0553 & 1,6582 & 1,6973 & 0,0391 & 0,0684 \\
262 & 1,2574 & 1,2486 & 0,0088 & 1,5057 & 1,4857 & 0,0200 & 1,6496 & 1,5766 & 0,0730 & 0,0762 \\
263 & 1,3159 & 1,3120 & 0,0039 & 1,4512 & 1,4046 & 0,0466 & 1,6659 & 1,7408 & 0,0749 & 0,0883 \\
264 & 1,2686 & 1,2722 & 0,0036 & 1,4776 & 1,4271 & 0,0505 & 1,5043 & 1,5046 & 0,0003 & 0,0506 \\
265 & 1,1716 & 1,1668 & 0,0048 & 1,4345 & 1,4118 & 0,0227 & 1,4608 & 1,4664 & 0,0056 & 0,0239 \\
266 & 1,2591 & 1,2500 & 0,0091 & 1,4459 & 1,4126 & 0,0333 & 1,6284 & 1,6075 & 0,0209 & 0,0404 \\
267 & 1,2452 & 1,2478 & 0,0026 & 1,4881 & 1,4676 & 0,0205 & 1,5739 & 1,5183 & 0,0556 & 0,0593 \\
268 & 1,4453 & 1,4403 & 0,0050 & 1,5571 & 1,5316 & 0,0255 & 1,8812 & 1,8237 & 0,0575 & 0,0631 \\
269 & 1,3108 & 1,2853 & 0,0255 & 1,6766 & 1,5668 & 0,1098 & 2,0818 & 2,2224 & 0,1406 & 0,1802 \\
270 & 1,3207 & 1,2792 & 0,0415 & 1,6012 & 1,5632 & 0,0380 & 1,7564 & 1,6771 & 0,0793 & 0,0972 \\
271 & 1,2541 & 1,2384 & 0,0157 & 1,5541 & 1,5222 & 0,0319 & 1,5471 & 1,6465 & 0,0994 & 0,1056 \\
272 & 1,3377 & 1,3026 & 0,0351 & 1,5686 & 1,5575 & 0,0111 & 1,7688 & 1,8355 & 0,0667 & 0,0762 \\
273 & 1,4291 & 1,4290 & 0,0001 & 1,6344 & 1,5358 & 0,0986 & 2,1542 & 2,0394 & 0,1148 & 0,1513 \\
\hline
\end{tabular}


pelo volume analisado, obtendo-se, assim, o número médio da CEP por $\mathrm{mm}^{3}$, apresentados na Tabela 2.

\section{Resultados e Discussão}

Examinando os resultados das tortuosidades expressos na Tabela 1, observou-se que, em alguns casos, as tortuosidades de uma amostra diferem significativamente quando considerados os sentidos positivo e negativo da direção de varredura. Isso decorre do fato do algoritmo reconstruir algumas trabéculas distintas em cada sentido, que depende da conectividade trabecular ocorrer da primeira fatia até a última, mas também da forma da estrutura trabecular, que pode apresentar maior ou menor número de discos ou hastes na direção de propagação do plano varredor. Recentemente Saha et al. (2010) propuseram uma técnica para classificação da distribuição trabecular em hastes e discos, o que possibilitará uma investigação mais apropriada sobre a influência dessas formas na tortuosidade.

Definindo-se o desvio das tortuosidades direcionais (DTD) como $\Delta \tau_{k}=\left|\tau_{+k}-\tau_{-k}\right|$, onde $k=x, y, z$, e $\sigma=\sqrt{\Delta \tau_{x}^{2}+\Delta \tau_{y}^{2}+\Delta \tau_{z}^{2}}$, é possível analisar a variação da tortuosidade de acordo com a direção e com a amostra. Calculando o DTD para a amostra 269 na direção $x$, por exemplo, obtém-se $\Delta \tau_{x}=0.1406$, enquanto para a amostra 273, na direção $z$, obtém-se $\Delta \tau_{z}=0,0001$; esses representam o maior e o menor valor do DTD entre todas as amostras. Os DTDs, apresentados na Tabela 1, juntamente com os valores de $\sigma$, indicam também que a rede trabecular apresenta certa anisotropia na tortuosidade, ou seja, a rede trabecular apresenta-se com uma orientação preferencial que difere da direção de propagação do plano de varredura. Nota-se que

Tabela 2. Número médio da CEP por $\mathrm{mm}^{3}$, para cada amostra. Table 2. Average number of CEP by $\mathrm{mm}^{3}$, for each sample.

\begin{tabular}{cc}
\hline Amostra & $C E P$ \\
\hline 254 & $-3,413$ \\
255 & $-1,520$ \\
256 & $-2,745$ \\
262 & $-4,075$ \\
263 & $-3,015$ \\
264 & $-1,458$ \\
265 & $-4,910$ \\
266 & $-5,130$ \\
267 & $-3,879$ \\
268 & $-0,337$ \\
269 & $-0,261$ \\
270 & $-0,816$ \\
271 & $-1,909$ \\
272 & $-2,037$ \\
273 & $-0,375$ \\
\hline
\end{tabular}

os menores DTDs ocorrem na direção $z$, que para as amostras significam a direção distal-proximal do rádio. Estes baixos valores de DTD são um indicativo de que a rede trabecular procura alinhar-se na direção na qual ela está submetida, com maior frequência, a tensões e compressões (Tabor, 2009). Isto vem ao encontro da nossa conjectura de que a tortuosidade deve desempenhar um papel importante na competência mecânica da estrutura trabecular e, por isso, deve ser detalhadamente investigada.

Analisando-se os valores das Tabelas 1 e 2 e considerando as representações volumétricas das amostras 266, 268 e 269 (Figuras 3a-c, respectivamente), nota-se que a amostra 266 apresenta uma boa conectividade trabecular e baixos DTDs; a amostra 268 apresenta conectividade e DTDs intermediários. Já a amostra 269 apresenta uma baixa conectividade em uma região da rede trabecular e valores elevados dos DTDs, em consequência das trabéculas que ainda estão conectadas apresentarem grandes distâncias geodésicas, fato este decorrente da existência de grandes cavidades medulares. O estudo da correlação entre a CEP na Tabela 2 e os valores de $\sigma$ para as amostras na Tabela 1, mostrou que $r=0,64$, o que indica uma correlação positiva moderada, em outros termos, uma correlação negativa entre a conectividade e $\sigma$ (anisotropia na tortuosidade).

Considerando apenas uma única trabécula das amostras 266 e 269, foram geradas suas propagações por conectividade. Para cada uma dessas trabéculas foi estimada a tortuosidade e verifica-se que, em virtude do maior número e volume das cavidades medulares na amostra 269, a tortuosidade da trabécula na direção $+z\left(\tau_{+z}=1,8377\right.$, Figura $\left.4 \mathrm{~b}\right)$ é bem maior do que aquela para a amostra 266, com maior conectividade da rede $\left(\tau_{+z}=1,1693\right.$, Figura $\left.4 a\right)$.

O desenvolvimento de técnicas para estimar a tortuosidade do osso canceloso ainda é algo bastante recente. Em estudo de Attenborough et al. (2005), a tortuosidade das cavidades medulares foi obtida
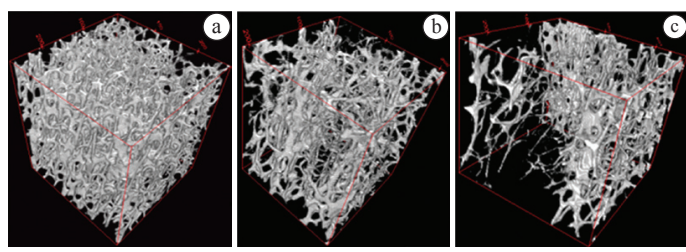

Figura 3. Visualizações 3D: a) amostra 266, com boa conectividade trabecular e baixo DTD; b) amostra 268, com conectividade e DTD intermediários; c) amostra 269, com grande cavidade medular, baixa conectividade e acentuado DTD.

Figure 3. 3D visualization: a) sample 266, with good connectivity and low DTD; b) sample 268, with intermediary connectivity and DTD; c) sample 269, with large marrow cavity, low connectivity and high DTD. 


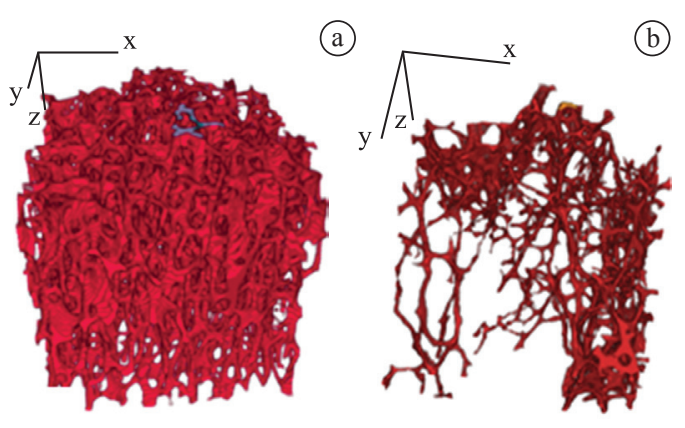

Figura 4. Conectividade por propagação a partir de uma única trabécula para amostras a) 266 e b) 269.

Figure 4. Connectivity by propagation for a single trabecula to samples a) 266 and b) 269 .

para amostras de réplicas do ilíaco, fêmur, vértebras lombares e calcâneo, utilizando pulsos de áudio frequência. No entanto, a estimativa da tortuosidade da rede trabecular apresentada neste trabalho e sua correlação com a conectividade é original. Embora os materiais, métodos e sítios das amostras tenham sido distintos, dificultando uma comparação mais efetiva entre os resultados, os valores apresentados na Tabela 1 estão consistentes com o intervalo de valores apresentados em Attenborough et al. (2005), quando considerada a dualidade entre cavidade medular e rede trabecular do osso canceloso a partir de imagens binarizadas.

\section{Conclusão}

A osteoporose causa a perda de massa óssea e a degradação da estrutura do osso trabecular, comprometendo sua resistência mecânica, por isso, estudar e compreender a microarquitetura trabecular é fundamental. Neste trabalho, a tortuosidade da rede trabecular foi estimada, através da técnica da reconstrução geodésica aplicada à fase sólida do osso canceloso nos seis sentidos do volume de 15 amostras do rádio distal. Os resultados obtidos deixam claro que há uma variação no grau de sinuosidade das trabéculas com relação à direção do plano varredor e isso leva a uma anisotropia na tortuosidade da rede. As menores anisotropias na tortuosidade ocorrem na direção $z$ que corresponde a direção distal-proximal do rádio. Este resultado é interessante e está de acordo com o conhecimento de que as trabéculas tendem a alinhar-se na direção de maiores tensões e compressões. Além disso, a tortuosidade se correlaciona à CEP de forma positiva moderada, o que sugere que estruturas melhor conectadas apresentam menor desvio das trabéculas em relação às direções de referência. Por outro lado, valores mais elevados de tortuosidade direcional parecem estar relacionados a uma maior ou menor existência de hastes ou discos na rede trabecular.
Os resultados desse trabalho são inovadores evidenciando uma correlação entre tortuosidade e conectividade trabecular. Claramente novos estudos devem ser conduzidos de forma a se obter uma melhor compreensão sobre o papel da tortuosidade como parâmetro estrutural da rede trabecular.

\section{Agradecimentos}

W. L. Roque agradece ao Dr. Z. Tabor pela concessão das imagens $\mu \mathrm{CT}$ das amostras e por inúmeros debates técnicos. K. Arcaro agradece à CAPES e R. Lanfredi agradece ao CNPq pelas bolsas de estudo concedidas.

\section{Referências}

Accardo AP, Strolka I, Toffanin R, Vittur F. Medical imaging analysis of the three dimensional (3D) architecture of trabecular bone: techniques and their applications. In: Leondes CT, editor. Medical Imaging Systems Technology - Methods in General Anatomy. Singapura: World Scientific Publishing Co. Pte. Ltd.; 2005. p. 1-41.

Araújo DV, Oliveira JH, Bracco OL. Custo da fratura osteoporótica de fêmur no sistema suplementar de saúde brasileiro. Arquivos Brasileiros de Endocrinologia \& Metabologia. 2005; 49(6):897-901.PMid:16544011.

Attenborough K, Shin H-C, Fagan MJ, Langton CM. Measurements of tortuosity in stereolithographical bone replicas using audiofrequency pulses (L). Journal of Acoustical Society of America. 2005; 118(5):2779-82. PMid:16334655. http://dx.doi.org/10.1121/1.2062688

Aygün H, Attenborough K, Postema M, Lauriks W, Langton CM. Prediction of angle dependent tortuosity and elasticity effects on sound propagation in cancellous bone. Journal of Acoustical Society of America. 2009; 126(6):3286-90. PMid:20000942. http://dx.doi.org/10.1121/1.3242358

Burge R, Dawson-Hughes B, Solomon DH, Wong JB, Kin A, Tosteson A. Incidence and economic burden of osteoporosis-related fractures in the United States: 2005-2025. Journal of Bone and Mineral Research. 2007; 22(3):465-75. PMid:17144789. http://dx.doi.org/10.1359/jbmr.061113

Carbonare LD, Valenti M, Bertoldo F, Zanatta M, Zenari S, Realdi G, Cascio VL, Giannini S. Bone microarchitecture evaluated by histomorphometry. Micron. 2005; 36(7-8):609-16. PMid:16242341. http:// dx.doi.org/10.1016/j.micron.2005.07.007

Chappard D, Legrand E, Pascaretti C, Baslé MF, Audran M. Comparison of eight histomorphometric methods for measuring trabecular bone architecture by image analysis on histological sections. Microscopy Research and Technique. 1999; 45(4-5):303-12. http://dx.doi.org/10.1002/ (SICI)1097-0029(19990515/01)45:4/5\%3C303::AID-JEM T14\%3E3.0.CO;2-8

Clennell MB. Tortuosity: a guide through the maze. In: Lovell MA, Harvey PK, editors. Developments in Petrophysics Geological Society Special Publication. London: Geological Society; 1997. v. 122, p. 299-344. 
Gommes CJ, Bons A-J, Blacher S, Dunsmuir JH, Tsou AH. Practical methods for measuring the tortuosity of porous materials from binary or gray-tone tomographic reconstructions. American Institute of Chemical Engineers Journal. 2009; 55(8):2000-12. http://dx.doi.org/10.1002/ aic. 11812

Gundersen HJG, Boyce RW, Nyengaard JR, Odgaard A. The connEulor: unbiased estimation of the connectivity using physical disectors under projection. Bone. 1993; 14(3):217-22. http://dx.doi.org/10.1016/8756-3282(93)90144-Y

Homminga J, McCreadie BR, Weinans H, Huiskes R. The dependence of the elastic properties of osteoporotic cancellous bone on volume fraction and fabric. Journal of Biomechanics. 2003; 36(10):1461-7. http://dx.doi. org/10.1016/S0021-9290(03)00125-8

Hughes ER, Leighton TG, White PR. Investigation of an anisotropic tortuosity in a Biot model of ultrasonic propagation in cancellous bone. Journal of Acoustical Society of America. 2007; 121(1):568-74. PMid:17297810. http://dx.doi.org/10.1121/1.2387132

Laib A, Beuf O, Issever A, Newitt DC, Majumdar, S. Direct measures of trabecular bone architecture from MR images. Advances in Experimental Medicine and Biology. 2001; 496:37-46.

Roberts N, Reed M, Nesbitt G. Estimation of the connectivity of a synthetic porous medium. Journal of Microscopy. 1997; 187(2):110-8. http://dx.doi.org/10.1046/ j.1365-2818.1997.2220784.x

Roque WL, Arcaro K, Freytag I. Tortuosidade da rede do osso trabecular a partir da reconstrução geodésica de imagens binárias tridimensionais. In: Workshop de Informática Médica: Anais do $11^{\circ}$ Workshop de Informática Médica; 2011 jul. 19-21; Natal, Brasil. CSBC; 2011. p. 1708-17.

Roque WL, Arcaro K, Tabor Z. An investigation of the mechanical competence of the trabecular bone. In: Dvorkin E, Goldschmit M, Storti M, editors. Mecánica Computacional. Buenos Aires: Editora AMCA; 2010. v. 29, p. 2001-9.
Roque WL, Souza ACA, Barbieri DX. The Euler-Poincaré characteristic applied to identify low bone density from vertebral tomographic images. Revista Brasileira de Reumatologia. 2009; 49(2):140-52. http://dx.doi.org/10.1590/ S0482-50042009000200006

Roque WL, Souza ACA, Barbieri DX, Rodrigues FC. Um sistema computacional baseado no processamento de imagens tomográficas para estudo da estrutura trabecular. In: Workshop de Informática Médica: Anais do $7^{\circ}$ Workshop de Informática Médica; 2007; Porto de Galinhas, Brasil. SBC; 2007. p. 156-65.

Saha PK, Xu Y, Duan H, Heiner A, Liang G. Volumetric topological analysis: A novel approach for trabecular bone classification on the continuum between plates and rods. IEEE Transactions on Medical Imaging. 2010; 29(11):1821-38. PMid:20562041. PMCid:3113685. http://dx.doi.org/10.1109/ TMI.2010.2050779

Tabor Z. On the equivalence of two methods of determining fabric tensor. Medical Engineering and Physics. 2009; 31(10):1313-22. http://dx.doi.org/10.1016/j. medengphy.2009.09.003

Vogel HJ, Kretzshmar A. Topological characterization of pore space in soil - sample preparation and digital image-processing. Geoderma. 1996; 73(1-2):23-38. http:// dx.doi.org/10.1016/0016-7061(96)00043-2

Wesarg S, Erdt M, Kafchitsas K, Khan MF. Direct visualization of regions with lowered bone mineral density in dual-energy CT images of vertebrae. In: Summers RM, Ginneken B, editors. The International Society for Optical Engineering - SPIE. Medical Imaging 2011: Proceedings of the Computer-Aided Diagnosis; 2011; Bellingham. Bellingham: SPIE Press; 2011. v. 12, p. 79633J-1-79633J-10. Part Two: Progress in Biomedical Optics and Imaging.

Wua YS, Van Vliet LJ, Frijlink HW, Van Der Voort Maarschalk $\mathrm{K}$.The determination of relative path length as a measure for tortuosity in compacts using image analysis. European Journal of Pharmaceutical Sciences. 2006; 28(5):433-40. PMid:16806859. http://dx.doi.org/10.1016/j.ejps.2006.05.006

\footnotetext{
Autores

Waldir Leite Roque*, Katia Arcaro, Ricardo Bigolin Lanfredi

Programa de Pós-Graduação em Matemática Aplicada, Instituto de Matemática - IM, Universidade Federal do Rio Grande do Sul - UFRGS, Rua Bento Gonçalves, 9500, Bairro Agronomia, CEP 91509-900, Porto Alegre, RS, Brasil.
} 